\title{
Hydrogen peroxide as a cause of iatrogenic subcutaneous cervicofacial emphysema: Is it innocent?
}

\author{
Mehmet Haksever, Davut Akduman \\ Department of Otorhinolaryngology, Bursa Sevket Yilmaz Education and Training Hospital, Bursa, Turkey \\ Email: drmehmethaksever@gmail.com
}

Received 7 June 2012; revised 9 July 2012; accepted 5 August 2012

\begin{abstract}
The subcutaneous cervicofacial emphysema is a rare and serious disease. There is a wide variety of etiologic factors for subcutaneous cervicofacial emphysema. As an maitrisable factor, the use of hydrogen peroxide $3 \%$ as an irrigant to newly operated tissue plans may cause emphysema. We report here a case with iatrojenic cervicofacial emphysema, occurred after the irrigation of hydrogen peroxide $3 \%$ to the drilled teeth root during dental treatment.
\end{abstract}

Keywords: Hydrogen Peroxide; Cervicofacial Emphysema; Dental Surgery

\section{INTRODUCTION}

Subcutaneous cervicofacial emphysema results from a wide variety of etiologies including trauma, surgery, dental procedures, tracheal intubation, pneumomediastinum and soft tissue infections [1]. Althougth it might be a self limiting condition, if enough air can pass through the deep space, it will cause pneumomediastinum resulting in the airway obsruction and of course superimposed infections [2,3]. The surgical intervention of molar teeth is the most frequent oral surgical procedure. Despite the beholding of surgical skills and being experienced, complications still may occur [4]. The high-speed drills, those are used during dental restoration, extraction and endodontic procedures are driven by a pressurized mixture of air and water. Also hydrogen peroxide 3\% (oxygenated water, $\mathrm{H}_{2} \mathrm{O}_{2}$ ) can be used for its anti-anaerobic activity, chemical cauterization of small vessels and effective irrigation of wounds. The patients with subcutaneous cervicofacial emphysema typically present with swelling and crepitus over the face and neck and significant tenderness. However subcutaneous cervicofacial emphysema can be misdiagnosed as a process of other diseases, such as angioedema, hematoma, superior vena cava syndrome or infections [1].

By the following report, we present a case with subcutaneous emphysema that occurred during sugical procedure of the second molar teeth, just after the irrigation of hydrogen peroxide 3\%. The management of the case is described and issues related to the diagnosis and prevention of cervicofacial emphysema are discussed.

\section{CASE REPORT}

A 47 years old female patient presented with her dentist to Sevket Yilmaz Education Training and Research Hospital, Otorhinolaryngology Department. The patient's medical history and information from the dentist revealed that she had undergone dental root treatment for upper left second molar tooth. Then progressive facial swelling and pain was noted on the left side just after the irrigation of hydrogen peroxide to the drilled root of tooth suddenly.

The patient arrived at our clinic 20 - 30 minutes later. She and her dentist were in a panic. On physical examination she had facial swelling, tenderness and crepitation on the left side of her face. The crepitus was also observed on her left eyelid and left side of the neck, but did not extend to below the clavicula. No significant other abnormalities were detected including airway problems, blood counts and coagulation screening tests.

The chest X-ray of patient commented normal. The paranasal computed tomography (PNCT) confirmed the presence of air within the subcutaneous soft tissues of the face, periorbita, masseteric space and infratemporal fossae (Figure 1). There was no air at mediastinum in thorax CT. The patient was hospitalised and screened with close-observation for possible pulmonary complications. She was given 1 gram ceftriaxone intravenous twice in a day during one week as prophylactic antimicrobial therapy. The emphysema gradually decreased and she was discharged to after 5 days.

\section{DISCUSSION}

The subcutaneous cervicofacial emphysema is an uncommon manifestation often associated with head and neck surgery [5], maxillofacial trauma [6], dental procedures [2,7], soft-tissue infections [3,7], tracheal intubation 

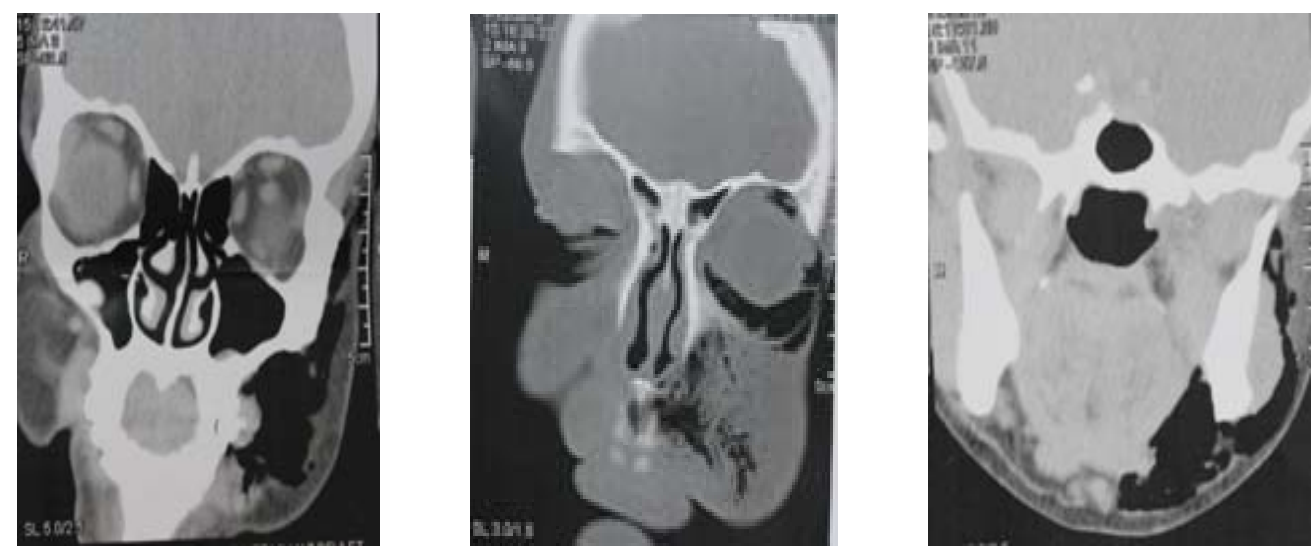

Figure 1. On coronal computed tomography facial swelling and air in subcutaneous facial tissue, eyelid and neck is seen.

[1,3], and any condition leading to pneumomediastinum [8]. McKenzie and Rosenberg [9], in a recent review, examined 32 cases with subcutaneous emphysema. Half of the cases were associated with the use of air-driven handpieces. Although air-driven handpieces were used in our case, subcutaneous cervicofacial emphysema was noted just after the irrigation of hydrogen peroxide $3 \%$ to the drilled root of tooth, as noted above. A comprehensive review of the literature shows that there are several cervicofacial emphysema cases following irrigation with hydrogen peroxide $3 \%[10,11]$. Now, it is possible to say that soluble gases in hydrogen peroxide $3 \%$ solution can disolve in bloody media and these gasses can pass through the newly damaged tissue planes resulting in emphysema.

In the most cases of subcutaneous cervicofacial emphysema, pneumomediastinum occurs as a result of air passage to the mediastinum by one of these three ways: 1) dissection along the fascial planes from either the neck or retroperitoneum; 2) air leakage by perforation of the trachea, bronchus or esophagus; 3) alveolar rupture and migration of air through the interstitium [12]. In our case pneumomediastinum did not developed but patient was followed for this potential complication.

The operation area of otolaryngology is adjacent to the dental region. Therefore using hydrogen peroxide $3 \%$ in oral-maxillofacial procedures has a potential risk for subcutaneous emphysema. There are many papers about the use of hydrogen peroxide 3\% in tonsillectomy operations to point out whether it decreases blood loss, pain and time of the operation $[13,14]$. Up to now there are no servicofacial emphysemas with the use of hydrogen peroxide $3 \%$ on tonsillectomy in our review of the literature. It may be supposed that, tonsillectomy and also some of other operations such as adenoidectomy, uvulopharingo palatoplasty, Caldwell-Luc procedure and other maxillofacial surgical interventions can damage the fascial tissue planes and using hydrogen peroxide 3\% for any reasons can cause cervicofacial emphysema, pneumome-diastinum and pneumo-retropharyngeum. Whenever the hydrogen peroxide $3 \%$ is used, pros and cons should be considered and the surgeon, the anaesthesists and postopretaive care unit nurses should be aware of this potential complication.

The management of cervicofacial emphysema should firstly include the evaluation of primary etiologic factor. The hospitalisation, the use of prophylactic antibiotics to reduce the risk of mediastinitis or deep neck infections and close observation to follow the complications are necessary. The administration of one hundred percent oxygen is told to increase the rate of gas resorption, It accelerates the treatment especially when pneumomediastinum develops. The use of laxative, cough supressant, analgesic and bed rest is part of the conservative therapy to eliminate the excessive intrathoracic pressure. The subcutaneous cervicofacial emphysema will spontaneously resolve over 1 to 2 weeks in the most of the unmcomplicated cases [12].

In conclusion, the cervicofacial emphysema is a uncommon but serious complication of dental and maxillofacial procedures. The use of hydrogen peroxide $3 \%$ as an irrigant may hazardously develop the cervicofacial emphysema in such surgical interventions. Although the treatment of cervicofacial emphysema is conservative, it should be aware of potential life threatening complications.

\section{REFERENCES}

[1] Goudarzi, M. and Navabi, J. (2011) Self-induced subcutaneous facial emphysema in a prisoner: Report of a case. Ear, Nose \& Throat Journal, 90, E5-E6.

[2] Chen, S.-C., Lin, F.-Y. and Chang, K.-J. (1999) Subcutaneous emphysema and pneumomediastinum after dental extraction. The American Journal of Emergency Medicine, 17, 678-680. doi:10.1016/S0735-6757(99)90158-4 
[3] Cavuslu, S., Oncul, O., Gungor, A., Kizilkaya, E. and Candan H. (2004) A case of recurrent subcutaneous emphysema as a complication of endotracheal intubation. Ear, Nose \& Throat Journal, 83, 485-488.

[4] Romeo, U., Galanakis, A., Lerario, F., Daniele, G.M., Tenore, G. and Palaia, G. (2011) Subcutaneous emphysema during third molar surgery: A case report. Brazilian Dental Journal, 22, 83-86.

[5] Marioni, G., De Filippis, C., Tregnaghi, A., Gaio, E. and Staffieri, A. (2003) Cervical emphysema and pneumomediastinum after tonsillectomy: It can happen. Otolaryngology_Head and Neck Surgery, 128, 298-300. doi:10.1067/mhn.2003.25

[6] Henry, C.H. and Hills, E.C. (1989) Traumatic emphysema of the head, neck, and mediastinum associated with maxillofacial trauma: Case report and review. Journal of Oral and Maxillofacial Surgery, 47, 876-882. doi:10.1016/S0278-2391(89)80053-9

[7] Frühauf, J., Weinke, R., Pilger, U., Kerl, H. and Müllegger, R.R. (2005) Soft tissue cervicofacial emphysema after dental treatment: Report of 2 cases with emphasis on the differential diagnosis of angioedema. Archives of Dermatology, 141, 1437-1440. doi:10.1001/archderm.141.11.1437

[8] López-Peláez, M.F., Roldán, J. and Mateo, S. (2001) Cervical emphysema, pneumomediastinum, and pneumotho- rax following self-induced oral injury: Report of four cases and review of the literature. Chest, 120, 306-309. doi:10.1378/chest.120.1.306

[9] McKenzie, W.S. and Rosenberg, M. (2009) Iatrogenic subcutaneous emphysema of dental and surgical origin: A literature review. Journal of Oral and Maxillofacial Surgery, 67, 1265-1268. doi:10.1016/j.joms.2008.12.050

[10] Kaufman, A.Y. (1981) Facial emphysema caused by hydrogen peroxide irrigation: Report of a case. Journal of Endodontics, 7, 470-472. doi:10.1016/S0099-2399(81)80309-3

[11] Singh, P.K. (2010) Root canal complications: The hypochlorite accident. Journal of the South African Dental Association, 65, 416-419.

[12] Ramchandar, K. and Carr, M.M. (2006) Spontaneous facial subcutaneous emphysema. Ear, Nose \& Throat Journal, 85, 744-746.

[13] Al-Abbasi, A.M. and Saeed, Z.K. (2008) Hydrogen peroxide 3\%: Is it beneficial in tonsillectomy? Sultan Qaboos University Medical Journal, 8, 201-204.

[14] Chacra, Z.A., Manoukian, J.J., Al-Qahtani, K., Al-Eisa, M., Balys, R., Hagr, A., Tewfik, T.L. and Schloss, M.D. (2005) Hydrogen peroxide mouth rinse: An analgesic post-tonsillectomy. The Journal of Otolaryngology, 34 178-182. doi:10.2310/7070.2005.04060 